\title{
Efeitos da posição sentada na força de músculos respiratórios durante 0 desmame de pacientes sob ventilação mecânica prolongada no pós-operatório de cirurgia cardiovascular
}

\author{
Effects of sitting position on the strength of respiratory muscles during weaning of patients \\ under prolonged mechanical ventilation in the postoperative period of cardiovascular surgery
}

Emília Nozawa ${ }^{1}$, Maria Ignez Zanetti Feltrim², Nidia Aparecida Hernandes ${ }^{3}$, Alessandra Preisig ${ }^{4}$, Luís Marcelo Sá Malbouisson ${ }^{5}$, José Otávio Costa Auler Júnior ${ }^{6}$

Estudo desenvolvido no InCor - Instituto do Coração do HCFMUSP - Hospital das Clínicas da Faculdade de Medicina da Universidade de São Paulo - São Paulo, SP, Brasil.

Fisioterapeuta; Doutora em Ciências pela Faculdade de Medicina da Universidade de São Paulo (USP); Chefe do Serviço de Fisioterapia do InCor do Hospital das Clínicas da Faculdade de Medicina da USP - São Paulo (SP), Brasil.

2 Fisioterapeuta; Doutora em Reabilitação pela Universidade Federal de São Paúlo (UNIFESP); Diretora do Serviço de Fisioterapia do Instituto do Coração (InCor) do Hospital das Clínicas da Faculdade de Medicina da Universidade de São Paulo (USP) - São Paulo (SP), Brasil.

Fisioterapeuta do Curso de Aprimoramento do InCor do Hospital das Clínicas da Faculdade de Medicina da USP - São Paulo (SP), Brasil.

${ }^{4}$ Fisioterapeuta do Curso de Especialização do InCor do Hospital das Clínicas da Faculdade de Medicina da USP - São Paulo (SP), Brasil.

Médico Assistente; Doutor do Serviço de Anestesiologia do Instituto Central do Hospital das Clínicas da Faculdade de Medicina da USP - São Paulo (SP), Brasil.

${ }^{6}$ Professor Titular de Anestesiologia da Faculdade de Medicina da USP; Diretor do Serviço de Anestesiologia do Incor do Hospital das Clínicas da Faculdade de Medicina da USP - São Paulo (SP), Brasil.

ENDERECO PARA CORRESPONDÊNCIA:

Emilia Nozawa - Av. Enéas de Carvalho Aguiar, 44 - CEP: 05403-000 - São Paulo (SP), Brasil-E-mail: fis_emilia@incor.usp. br

APRESENTAÇÃO jun. 2010

ACEITO PARA PUBLICACÃO fev. 2011

FONTE DE FINANCIAMENTO: Fundação EJ Zerbini

CONFLITO DE INTERESSE: nada a declarar
RESUMO: O objetivo do estudo foi investigar os efeitos da posição sentada, nos parâmetros ventilatórios e hemodinâmicos, em pacientes com suporte ventilatório mecânico prolongado, estáveis hemodinamicamente. Participaram do estudo 40 pacientes que foram randomizados em grupo controle $(n=17)$ e grupo intervenção $(n=23)$. Foram mensurados parâmetros hemodinâmicos, gasometria arterial, força muscular respiratória e ventilometria, realizados em dois momentos: primeira e segunda avaliação, com intervalo de 30 minutos entre as medidas. No grupo controle, as duas avaliações foram realizadas no leito, com a cabeceira elevada a $30^{\circ}$. No grupo intervenção, a primeira avaliação foi realizada no leito $\left(30^{\circ}\right)$ e a segunda, 30 minutos após transferência para a poltrona, na posição sentada $\left(90^{\circ}\right)$. A idade média da amostra foi de $64,7 \pm 11,2$ anos. O resultado do estudo demonstrou que não houve diferenças em relação às variáveis, hemodinâmicas, gasométricas, capacidade vital forçada, volume minuto e volume de ar corrente. Entretanto, ocorreu aumento significativo da pressão inspiratória máxima (PImáx) no grupo intervenção $(p<0.01)$. Concluímos que pacientes em pós-operatório de cirurgia cardiovascular em ventilação mecânica podem se beneficiar da posição sentada durante o desmame do suporte ventilatório, observado pela melhora da força muscular inspiratória (PImáx). Além disso, não foram observadas alterações hemodinâmicas com a troca de postura, sendo considerado um procedimento seguro.

Descritores: cirurgia torácica; ventilação mecânica; desmame; posicionamento do paciente.

ABSTRACT: The purpose was to study the effects of sitting position, in ventilatory and hemodynamic parameters, in patients under prolonged mechanical ventilation, in the postoperative of cardiovascular surgery. Participated 40 postoperative cardiovascular patients, intubated and mechanically ventilated, were randomized into control group $(n=17)$ and intervention group $(n=23)$. Hemodynamic parameters, arterial blood gases, respiratory muscle strength and ventilometry, were measured in two moments: first and second evaluation, with 30-minute interval between measurements. In the control group, both evaluations were performed at head of the bed elevated at $30^{\circ}$. In the sitting group, the first evaluation was developed at $30^{\circ}$ and the second, $(30$ minutes after transfer to the armchair) in the sitting position $\left(90^{\circ}\right)$. The mean age of patients was $64.7 \pm 11.2$ years. There were no differences regarding the hemodynamic parameters, blood gas analysis, forced vital capacity, minute ventilation and tidal volume. However, a significant increase in maximal inspiratory pressure (MIP) in the intervention group was observed $(p<0.01)$. We conclude that stable patients in the postoperative period of cardiovascular surgery, mechanically ventilated, maybe benefits of the sitting position observed of increased inspiratory muscle strength. Furthermore, no significant hemodynamic changes, and it was well tolerated when the patients were transferred to the sitting position, so it was considered a safe procedure.

KeYwords: thoracic surgery; artificial respiration; weaning; patient positioning. 


\section{INTRODUCÃO}

A cirurgia cardiovascular evoluiu muito nas últimas décadas, principalmente com a realização de alguns procedimentos sem necessidade de circulação extracorpórea (CEC), melhor proteção miocárdica, avanço nos métodos anestésicos e melhora dos cuidados intensivos, como a extubação precoce (fast track $)^{1}$. Mesmo assim, alguns pacientes permanecem por tempo prolongado em ventilação mecânica, com consequente aumento da incidência de complicações respiratórias e dos custos hospitalares. Dessa forma, esforços adicionais são necessários para auxiliar no desmame da ventilação mecânica nesses casos ${ }^{2}$. Os pacientes submetidos à cirurgia cardíaca permanecem, primariamente, na posição supina, intubados e sob ventilação mecânica e por longos períodos o que pode favorecer a ocorrência de pneumonia associada à ventilação mecânica e maior tempo de permanência na UTI. Efeitos deletérios da imobilização podem ser minimizados com técnicas fisioterapêuticas utilizadas no manuseio destes pacientes, incluindo posicionamento e mobilização ${ }^{3-5}$.

Estudos em indivíduos saudáveis têm demonstrado efeitos benéficos da posição sentada quando comparado com a posição supina, pois essa postura melhora a capacidade residual funcional (CRF), o volume de ar corrente (VC), o volume residual (VR) e o volume de reserva expiratório (VRE). A verticalização do corpo promove redução das forças gravitacional, mecânica e de compressão sobre os pulmões, sobre a parede torácica e sobre o diafragma ${ }^{6-8}$, com melhora da expansão ântero-posterior do tórax.. Este efeito é potencializado pelo encorajamento do próprio paciente a respirar espontânea e profundamente, aumentando assim a ventilação alveolar ${ }^{9}$.

Apesar de alguns estudos demonstrarem os benefícios da posição sentada em relação à posição supina em indivíduos saudáveis ${ }^{4,9}$ e em pacientes cardiopa$\operatorname{tas}^{10}$ ou mesmo em pacientes de pósoperatório, em ventilação espontânea ${ }^{6}, \mathrm{o}$ uso desse posicionamento em pacientes em ventilação mecânica invasiva após cirurgia cardiovascular, ainda necessita de maiores estudos. O objetivo foi comparar os parâmetros ventilatórios, de troca gasosa, hemodinâmicos e de força muscular respiratória com a troca da posição supina $\left(30^{\circ}\right)$ para a posição sentada $\left(90^{\circ}\right)$ em pacientes estáveis e em processo de desmame da ventilação mecânica no pós-operatório de cirurgia cardiovascular.

\section{CASUÍSTICA E MÉTODOS}

Foi conduzido um ensaio clínico randomizado e controlado. A amostra foi composta por 40 pacientes de pósoperatório de cirurgia cardiovascular, intubados e que não evoluíram dentro do protocolo de desmame e permaneceram por mais de 48 horas sob ventilação mecânica. Os pacientes foram randomizados em dois grupos: Controle (C) e Intervenção (I), através de um programa computacional de análise estatística.

Foram incluídos indivíduos adultos, de pós-operatório de cirurgia de revascularização do miocárdio, cirurgia valvar ou correção de aneurisma de aorta, ainda intubados, com mais de 48 horas de ventilação mecânica, estabilidade hemodinâmica, nível de consciência preservado (alerta e colaborativo) e em processo de desmame do suporte ventilatório. Foram excluídos os pacientes que apresentavam contraindicação para sair do leito (uso de balão intra-aórtico, cateter na artéria femoral) e caso recusassem em colaborar com o estudo. Todos os pacientes ou responsáveis assinaram um Termo de Consentimento Livre e Esclarecido aprovado pela Comissão de Ética para análise de Projetos de Pesquisa (CAPPesq) do Hospital das Clinicas da Faculdade de Medicina da Universidade de São Paulo sob o protocolo de pesquisa no 173/05.

Foram mensurados parâmetros hemodinâmicos, gasometria arterial, força muscular respiratória e ventilometria, realizados em dois momentos: primeira e segunda avaliação, com intervalo de 30 minutos entre as medidas. No grupo controle, as duas avaliações foram realizadas no leito, com a cabeceira elevada a $30^{\circ}$. No grupo intervenção, a primeira avaliação foi realizada no leito $\left(30^{\circ}\right)$ e a segunda, 30 minutos após transferência para a poltrona, na posição sentada $\left(90^{\circ}\right)$.

Foram registrados os parâmetros ventilatórios, como modalidade, freqüência respiratória (FR), volume corrente exalado (VC), pico de pressão inspiratória (PPI), pressão expiratória positiva final
(PEEP), pressão de suporte (PS), fração inspirada de oxigênio $\left(\mathrm{FiO}_{2}\right)$, índice de oxigenação (relação $\mathrm{PaO}_{2} / \mathrm{FiO}_{2}$ ), saturação periférica de oxigênio $\left(\mathrm{SpO}_{2}\right)$, índice de respiração rápida e superficial (relação FR/VC). Dentre os parâmetros hemodinâmicos, foram analisados: freqüência cardíaca (FC), pressão arterial sistólica (PAS), pressão arterial diastólica (PAD) e pressão arterial média (PAM).

A força muscular respiratória foi avaliada por meio da mensuração das pressões inspiratória e expiratória máximas (PImáx e PEmáx, respectivamente) utilizando-se um manovacuômetro $\left(\right.$ Marshall Town $\left.{ }^{\circledR}\right)$, conectado diretamente na tubo endotraqueal do paciente. A PImáx foi realizada solicitando-se ao paciente uma inspiração voluntária máxima a partir da CRF. Na aferição da PEmáx, o paciente foi orientado a realizar uma expiração máxima, também, a partir da CRF. Cada manobra foi repetida três vezes, sendo anotado o maior valor. Caso este ocorresse na última manobra, o teste era prosseguido até que fosse produzido um valor menos elevado ${ }^{11}$.

A CV e o VM foram medidos através de um ventilômetro (Ferraris ${ }^{\circledR} /$ Mark 8), conectado diretamente na cânula orotraqueal do paciente. Para mensuração da $C V$, solicitou-se ao paciente inspirar até a capacidade pulmonar total (CPT) e, em seguida, expirar tão forte e rápido possível até o volume residual. A manobra foi repetida três vezes com registro do maior valor. OVM foi mensurado a partir de respirações espontâneas registradas durante o período de um minuto ${ }^{12}$.

Para avaliação do risco pré-operatório e do índice de gravidade foram utilizados os escores de estratificação Parsonnet ${ }^{13} \mathrm{e}$ APACHE II ${ }^{14}$, respectivamente. Mudanças nas doses ou concentração de drogas vasodilatadores ou medicações inotrópicas positivas foram registradas. Analgésicos foram administrados de acordo com a prescrição médica.

O desmame da ventilação mecânica ocorreu de acordo com o protocolo padronizado na Unidade de Recuperação Cirúrgica até atingir os seguintes parâmetros: modo ventilatório SIMV (ventilação mandatória sincronizada intermitente) com $F R=02$ respirações por minuto, $\mathrm{PEEP}=5 \mathrm{cmH}_{2} \mathrm{O}, \mathrm{PS}=10$ $\mathrm{cmH}_{2} \mathrm{O}, \mathrm{FiO}_{2}=0,4$, PImáx $=-25 \mathrm{cmH}_{2} \mathrm{O}$, 
índice de oxigenação $\left(\mathrm{PaO}_{2}, \mathrm{FiO}_{2}>200\right)$, FR/VC $<105$, mantendo $\mathrm{SpO}_{2} \geq 95 \%$. Não houve qualquer alteração dos parâmetros ventilatórios ajustados no ventilador entre as medidas, em ambos os grupos.

\section{Análise estatística}

As variáveis quantitativas foram descritas em média e desvio padrão e frequências absolutas e relativas para as variáveis qualitativas. As médias dos grupos foram comparadas por meio do teste t de Student e teste paramétrico de MannWhitney. Para a análise dos dois grupos ao longo do tempo utilizou-se o teste de Análise de Variância com Medidas Repetidas. Os dados foram analisados no programa estatístico SPSS e as diferenças foram consideradas estatisticamente significativas para um alpha de 5\%.

\section{RESULTADOS}

A amostra foi composta de 42 pacientes adultos, sendo que dois pacientes foram excluídos do protocolo por falta de colaboração nas medidas da $2 \underline{a}$ avaliação. Dos 40 pacientes remanescentes, 26 eram do sexo masculino, sendo 13 do grupo controle e 13 do grupo intervenção. As características basais dos pacientes foram similares nos dois grupos e estão demonstradas na Tabela 1. Os procedimentos cirúrgicos realizados são descritos na Tabela 2.

Não houve diferenças em relação aos parâmetros hemodinâmicos ao longo do tempo nos dois grupos. Além disso, não foi necessário o ajuste de drogas vasoativas durante o estudo, embora 52\% dos pacientes do grupo controle e $61 \%$ do grupo intervenção necessitassem de alguma droga vasopressora (Tabela 3).

Em relação às medidas de força muscular respiratória, ocorreu aumento da PImáx no grupo que foi transferido para a posição sentada (I) quando comparado com o grupo que foi mantido em decúbito dorsal (C) (Tabela 3 e Figura 1). Em relação à PEmáx, houve tendência de aumento dessa variável na posição sentada, porém, sem diferença estatística. Os demais parâmetros analisados não sofreram alterações significativas com a troca de postura e são descritos na Tabela 3.
Tabela 1. Características da amostra

\begin{tabular}{lccc}
\hline Parâmetros & Controle & Sentado & Valores $\mathbf{p}$ \\
\hline Idade (anos) & $62,9 \pm 12.7$ & $61,5 . \pm 12,3$ & $\mathrm{NS}$ \\
Sexo $(\mathrm{M} / \mathrm{F})$ & $13 / 17$ & $13 / 23$ & $\mathrm{NS}$ \\
IMC $\left(\mathrm{Kg} / \mathrm{m}^{2}\right)$ & $27,0 \pm 5,8$ & $26,9 \pm 5,2$ & $\mathrm{NS}$ \\
Escore de risco cirúrgico & $20 \pm 10$ & $18 \pm 8$ & $\mathrm{NS}$ \\
APACHE II & $8,4 \pm 3.0$ & $9,6 \pm 2.7$ & $\mathrm{NS}$ \\
Tempo de cirurgia (min) & $393,5 \pm 110.5$ & $333,8 \pm 53,3$ & $\mathrm{NS}$ \\
Tempo de circulação & $130,4 \pm 59,0$ & $116 \pm 47,0$ & $\mathrm{NS}$ \\
extracorpórea (min) & & & \\
Tempo em ventilação & $73 \pm 79$ & $103 \pm 80$ & $\mathrm{NS}$ \\
mecânica (horas) & & & \\
\hline
\end{tabular}

IMC: índice de massa corporal; APACHE II: Acute Physiology and Chronic Health Evaluation; NS: sem diferença estatística entre os grupos

Tabela 2. Procedimentos operatórios

\begin{tabular}{lccc}
\hline Variáveis & Controle & Sentado & Total \\
\hline Operação valvar & 6 & 5 & 11 \\
RM & 6 & 3 & 9 \\
RM+ operação valvar & 3 & 7 & 10 \\
Correção de aneurisma de aorta & 2 & 3 & 5 \\
RM+ Correção de aneurisma de aorta & - & 5 & 5 \\
Total & 17 & 23 & 40 \\
\hline
\end{tabular}

RM: cirurgia de revascularização do miocárdio

Tabela 3. Variáveis respiratórias e de oxigenação nas posições supino e sentado.

\begin{tabular}{lcccc}
\hline Variável & \multicolumn{2}{c}{ Controle } & \multicolumn{2}{c}{ Intervenção } \\
\hline & supino & supino & supino & sentado \\
\hline Plmáx & $36 \pm 11$ & $39 \pm 12$ & $37 \pm 11$ & $46 \pm 11^{*}$ \\
PEmáx & $48 \pm 19$ & $56 \pm 23$ & $49 \pm 20$ & $60 \pm 21$ \\
$\mathrm{FC}(\mathrm{bpm})$ & $97 \pm 17$ & $97 \pm 17$ & $97 \pm 16$ & $95 \pm 19$ \\
$\mathrm{PAM}(\mathrm{mmHg})$ & $87 \pm 15$ & $88 \pm 10$ & $87 \pm 13$ & $88 \pm 13$ \\
$\mathrm{PaCO}_{2}(\mathrm{mmHg})$ & $39 \pm 5$ & $38 \pm 7$ & $38 \pm 4$ & $38 \pm 4$ \\
$\mathrm{SaO}_{2}(\%)$ & $97 \pm 1$ & $96 \pm 4$ & $96 \pm 2$ & $96 \pm 4$ \\
$\mathrm{PaO}_{2} / \mathrm{FlO}$ & $257 \pm 56$ & $260 \pm 55$ & $246 \pm 71$ & $246 \pm 60$ \\
$\mathrm{CVF} / \mathrm{l} / \mathrm{min}$ & $2,24 \pm 0,6$ & $2,14 \pm 0,7$ & $2,13 \pm 0,4$ & $2,14 \pm 0,7$ \\
$\mathrm{VM}(\mathrm{l} / \mathrm{min})$ & $11,2 \pm 1$ & $10,8 \pm 1$ & $11,3 \pm 3$ & $11,7 \pm 3$ \\
$\mathrm{VC}(\mathrm{ml})$ & $528 \pm 117$ & $532 \pm 116$ & $456 \pm 120$ & $484 \pm 130$ \\
$\mathrm{FR}(\mathrm{rpm})$ & $17 \pm 4$ & $17 \pm 5$ & $18 \pm 6$ & $18 \pm 7$ \\
\hline IRRS & $33 \pm 18$ & $37 \pm 24$ & $37 \pm 24$ & $38 \pm 20$ \\
\hline
\end{tabular}

Pimáx: pressão inspiratória máxima, PEmáx: pressão expiratória máxima, FC: frequência cardíaca, PAM: pressão arterial média, $\mathrm{PaCO}_{2}$ : pressão parcial de gás carbônico no sangue arterial, $\mathrm{SaO}_{2}$ : saturação de oxihemoglobina arterial, $\mathrm{PaO}_{2}$ : pressão parcial de oxigênio no sangue arterial, $\mathrm{PaO}_{2} / \mathrm{FIO}_{2}$ : índice de oxigenação, CVF: capacidade vital forçada, VM: volume minuto, VC: volume de ar corrente, FR: frequência respiratória, IRRS: índice de respiração rápida superficial $* p<0,05$ 


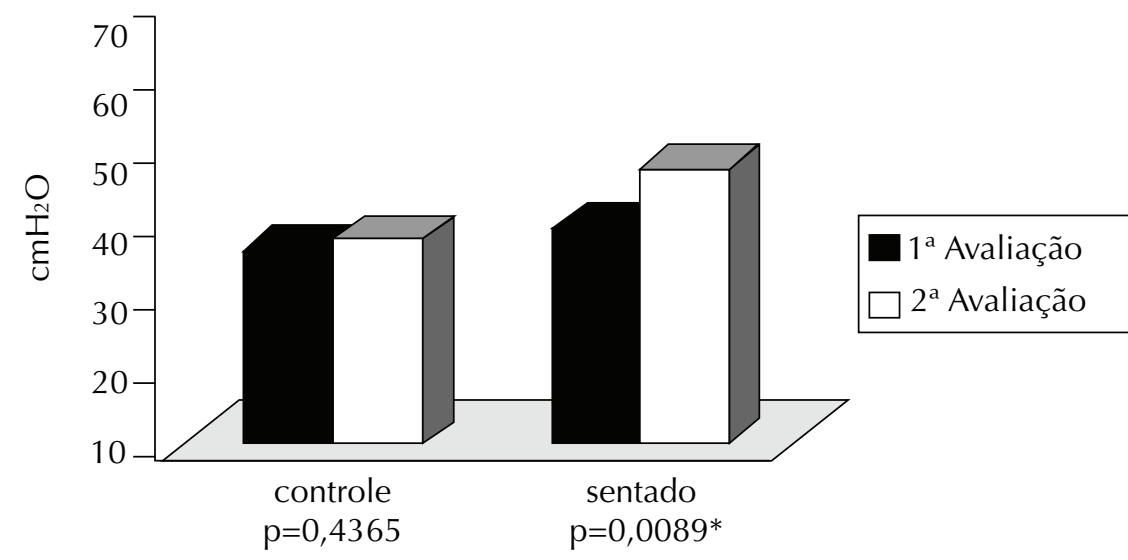

Figura 1. Valores médios da pressão inspiratória máxima do grupo controle e sentado

$* \mathrm{p}<0,05$

DISCUSSÃO

Nosso estudo comparou a posição de decúbito dorsal com a posição sentada na poltrona em pacientes de pós-operatório de cirurgia cardíaca, ainda sob ventilação mecânica, mas estáveis e em processo de desmame. Observamos aumento significativo da Plmáx na posição sentada quando comparada com a supina. Possivelmente, o aumento dessa variável está relacionado com melhor acoplamento toraco-abdominal e com o posicionamento do coração e do mediastino sobre os pulmões. Pacientes com tempo prolongado de ventilação mecânica apresentam redução da Pimáx ${ }^{15,16}$ e o sucesso no desmame está relacionado com valores adequados dessa medida, sendo essa variável considerada de alta sensibilidade e especificidade como preditor de sucesso no desmame ${ }^{17}$. Embora as medidas das pressões inspiratórias máximas sejam de simples realização e bem toleradas pelos pacientes, apresentam como desvantagens a necessidade de total colaboração. Este fator torna-se mais importante se considerarmos a presença do tubo traqueal que impõe certa dificuldade, o que justifica as baixas medidas que encontramos. As pressões medidas durante as manobras refletem as pressões desenvolvidas pelos músculos, somada a pressão de recolhimento elástico do sistema respiratório, que varia de acordo com o volume pulmonar. Como a relação entre a capacidade vital e a PImáx é curvilínea, variações na Plmáx podem ocorrer antes que alterações no volume pulmonar possam ser identificados. As pressões respiratória máximas (PImáx e PEmáx) foram realizadas ao nível da capacidade residual funcional.
A escolha deste método justificou-se pela presença do tubo endotraqueal que impõe resistência considerável aos fluxos inspiratório e expiratório e aumenta o espaço morto. Em nosso meio Neder e cols. ${ }^{18}$, Costa et al. ${ }^{19}$ propuseram equações para valores de referência para a população brasileira, a partir das medidas realizadas em volumes pulmonares extremos (volume residual para PImax e capacidade pulmonar total para PEmax), em posição sentada para $90^{\circ}$. Nestes dois estudos, gênero e idade foram as variáveis de maior poder preditivo. Nossos pacientes realizaram as medidas pressóricas a partir da CRF e em posicionamento de $30^{\circ}$ considerado como dorsal , e a $90^{\circ} \mathrm{em}$ outra medida, não sendo possível aplicar as equações preditoras. Por outro lado, as medidas, principalmente da PImáx, em pacientes intubados, propõemse a servirem como parâmetro para a extubação, sendo os valores como -25 a -30 $\mathrm{CmH}_{2} \mathrm{O}$ utilizados para este fim.

O posicionamento em sedestação aumenta o volume pulmonar ${ }^{20,21}$ e esse fato pode determinar o aumento da força dos músculos respiratórios. Um estudo avaliou a PImáx na posição supina, comparando com a posição sentada em pacientes com doença neuromuscular. Os pacientes foram divididos em dois grupos: com e sem respiração paradoxal e os autores verificaram que, em ambos os grupos, houve melhor performance na posição sentada quando comparado com a posição supina ${ }^{22}$.

Em nosso estudo, a PEmáx apresentou tendência ao aumento na posição sentada. É provável que essa variação esteja relacionada à vantagem mecânica da caixa torácica, nessa posição, onde ocorre aumento do tônus dos músculos expiratórios, que pode contribuir para o aumento da pressão abdominal durante a manobra ${ }^{23}$.

Não observamos qualquer efeito adverso da freqüência cardíaca (FC) e/ou da pressão arterial média (PAM) quando os sujeitos passaram da posição supina para sentada na poltrona. Apesar de mais da metade da amostra fazer uso de drogas vasoativas, não houve necessidade de aumento das dosagens durante a mudança de posição, demonstrando ser um procedimento seguro. Estudos com pacientes em ventilação mecânica invasiva não observaram mudanças no débito cardíaco com a troca da posição supina para a lateral $^{21,}$ e da FC e da PAM da posição supina para a posição sentada ${ }^{24}$.

Consideramos este estudo importante, pois possibilita a utilização de uma técnica fisioterapêutica que é o posicionamento corporal, na otimização do desmame prolongado e difícil em pacientes de alta complexidade.

\section{Limitações do estudo}

Nosso estudo foi conduzido em uma amostra de 40 pacientes com dificuldade na supressão da ventilação mecânica. Este tamanho amostral nos impede de outras afirmações conclusivas e, talvez, tenha sido insuficiente para revelar outras alterações que pudessem estar ocorrendo. A PImáx é uma medida volume dependente. Embora a CV e VC tenham sido monitorados eles não foram suficientes para apresentarem alterações significantes com a mudança de decúbito. Provavelmente, outras medidas como a capacidade inspiratória, tivessem maior sensibilidade.

\section{CONCLUSÃO}

Pacientes em pós-operatório de cirurgia cardiovascular com mais de 48 horas em ventilação mecânica podem se beneficiar da posição sentada durante o desmame do suporte ventilatório, observado pela melhora da força muscular inspiratória (PImáx). Este procedimento demonstrou ser seguro, pois não houve alterações hemodinâmicas durante a troca de postura nos pacientes estudados. 


\section{REFERÊNCIAS}

1. Szeles TF, Yoshinaga EM, Alencar W, Brudniewski M, Ferreira FS, Auler Jr JOC, MJC Carmona, Malbouisson LMS. Hypoxemia after Myocardial Revascularization: Analysis of Risk Factors. Rev Bras Anestesiol. 2008;58(2):124-36.

2. Trouillet JL, Combes A, Vaissier E, Luyt CE, Ouattara A, Pavie A, Chastre J. Prolonged mechanical ventilation after cardiac surgery: outcome and predictors. J Thorac Cardiovasc Surg. 2009;138(4):948-53.

3. Banasik JL, Bruya MA, Steadman RE, Demand JK, Effect of position on arterial oxygenation in postoperative coronary revascularization patients. Heart \& Lung. 1987;16(6):652-7.

4. Dean E. Effect of body position on pulmonary function. Physical Therapy. 1985;65(5):613-8.

5. Draculovic MB, Torres A,Bauer TT, Nicolas JM, Nogué S, Ferrerm.Supine body position as a risk factor for nosocomial pneumonia in mechanically ventilated patients: a randomised trial. The Lancet. 1999;354:1853-6.

6. Behrakis PK, Baydur A, Jaeger MJ, Emili JM. Lung mechanics in sitting and horizontal body positions. Chest. 1983;83:643-6.

7. Takazakura R, Takahashi M, Nitta N, Murata K. Diaphragmatic motion in the sitting and supine positions: healthy subject study using a vertically open magnetic resonance system. J Mag Res Imag. 2004;19:605-9.

8. Barnas GM, Green MD, Mackenzie CF, Fletcher SJ, Campbell N, Runcie C, Broderick GE. Effects of posture on lung and regional chest wall mechanics. Anestehesiology. 1993;78(2):251.

9. Jones AY, Dean E. Body position change and its effect on hemodynamic and metabolic status. Heart \& Lung. 2004;33:281-90.

10. Yasuda N, YagiY, Nagashima K, Sawa T, Nomura M, Hirakawa S, Figiwara H. Mechanism of posturally induced crackles as predictor of latent congestive heart failure. Respiration. 1997;64(5):336-41.

11. ATS/ERS (American Thoracic Society/European Society). Statement on Respiratory Muscle Testing. 10. Assessment of Respiratory Muscle Function in the Intensive Care Unit. Am J Respir Crit Care Med. 2002;166:610-24.

12. Matews PJ, Gregg BL. Monitorização e Controle do Paciente na UTI. In: Scanlan CL, Wilkins RL, Stoller JK. Fundamentos da Terapia Respiratória de Egan. $7^{\text {th }}$ ed. São Paulo: Manole. 2000; 975.
13. Parsonent $V$, Dean D, Berstein AD. A method of uniform stratification of risk for evaluating the results of surgery in acquired adult heart disease. Circulation. 1989; 79(suppl I): I-3-I12.

14. Knaus WA, Drapper EA, Wagner DP, Zimmerman JE. APACHE II:a severity of desease classificationsystem. Crit Care Med. 1985;13:818-29.

15. Chang AT, Boots RJ, Brown MG, Paratz J, Hodges EW. Reduced inspiratory muscle endurance following successful weaning from prolonged mechanical ventilation. Chest. 2005;128(2):553-9.

16. Bruton A. A pilot study of investigate any relationship between sustained maximal inspiratory pressure and extubation outcome. Heart \& Lung. 2002;31(2):141-9.

17. Badr C, Elkins MR, Ellis ER. The effect of body position on maximal expiratory pressure and flow. Oust J Physiol. 2002;48:95-102.

18. Neder J.A., Andreoni S, Lerario MC,Nery LE. Reference values for lung function tests.II. Maximal respiratory pressures and voluntary ventilation Braz J Med Res. 1999;32(6):719-27.

19. Costa D, Gonçalves HÁ, Lima LP, Ike D, Canciliero MK, Montebelo MIL. Novos valores de referência para pressões respiratórias máximas na população brasileira. J Bras Pneum. 2010;36(3):306-12.

20. Talwar A, Sood S, Sethi J. Effect of body posture on dynamic lung functions in young non-obese Indian subjects. Ind J Med Sci. 2002;56(12):607-12.

21. Nielsen KG, Holte K, Kehlet H. Effects of posture on postoperative pulmonary function. Acta Anaesthesiol Scand. 2003;47:1270-5.

22. Fromageot $C$, Lofaso F, Annane D Supine fall in lung volumes in the assessment of diaphragmatic weakness in neuromuscular disorders. Arch Phys Med Rehabil. 2001;82:123-8.

23. Laat E, Schoomnhovem L, Grypdonck M, Verbeek A, Graaf R, Pickkers P, Achterberg T. Early postoperative $30^{\circ}$ lateral positioning after coronary artery surgery: influence on cardiac output. Journal of Clinical Nursing. 2007;654-61.

24. Porto EF, Castro AAM, Leite JRO, Miranda SV, Lancauth A, Kumpel C. Análise comparativa da complacência do sistema respiratório em três diferentes posições no leito (lateral, sentado e dorsal) em pacientes submetidos à ventilação mecânica prolongada. Rev Bras Ter Intensiva. 2008;20(3):213-9. 